\title{
Mobile Technology System Ffor Health Intervention (M-Health)
}

\author{
Aadeesh Jain, Shruti Sabherwal, Deepankar Mathur, Meenakshi Sood
}

\begin{abstract}
The developments in the new technologies have made a huge impact in the field of health care. One such technology is mobile health system. There has been tremendous increase in the research regarding the potential of mobile health systems. Due to this increase there have been a lot of attempts to analyse and record the benefits of mobile health, focusing mainly on mobile health devices and state of health of a person. In this study, we combined an Arduino microcontroller with an Androidbased Smartphone to design a temperature and a heart rate module. The AD8232 Single Lead Heart Rate Monitor, Pulse sensor and LM35 temperature sensor is the centerpiece of this design. The information is transferred to mobile phones using Bluetooth which is obtained on an app created using MIT App Inventor 2. The data received is utilized to monitor the heart rate of a person in beats per minute as well as temperature in degree Celsius. The received data can be used to analyze the health conditions of the person under observation.

Index Terms: Pulse rate, Pulse, Temperature, Microcontroller, Bluetooth, Smartphone.
\end{abstract}

\section{INTRODUCTION}

The growth in computing power and mobile connectivity has created a foundation for mobile health (M-Health) technology that can transform the quality of health care on a large scale. Mobile health system is defined as usage of mobile devices to collect data from patients and storing the information on network servers. The database containing information about patients can then be easily accessed by doctors, medical staff, physicians, pharmacists, specialist etc. The incorporation of mobile health devices is important in obtaining information immediately to diagnose illness, track diseases and provide real time information [1]. The improvement in technology as well as wireless communication offers the potential for smart miniature devices as well as wearable detection, processing and communication.

Internet of things (IOT) is a system of connecting physical objects such that data can be transferred between them. IOT is majorly used in the healthcare sector. The mobilization of sensors used for medical purposes along with connecting devices is the crucial parts of mobile health system and IOT. These smart gadgets can be used to measure various criteria for example, temperature, diabetes, pulse rate etc. The mobile health data can be analyzed at present by using intelligent

Revised Manuscript Received on April 12, 2019. University of Information Technology, Solan, Himachal Pradesh, India

Shruti Sabherwal, Electronic and Communication Engineering, Jaypee University of Information Technology, Solan, Himachal Pradesh, India.

Deepankar Mathur, Electronic and Communication Engineering, Jaypee University of Information Technology, Solan, Himachal Pradesh, India.

Meenakshi Sood, Electronic and Communication Engineering, Jaypee University of Information Technology, Solan, Himachal Pradesh, India.
Aadeesh Jain, Electronic and Communication Engineering, Jaypee

algorithms which raises different alert levels depending upon the condition of the patient.

The previous researches indicate that a tool can be used to monitor real time pulse rate as well as the temperature of the patient without actually going to the doctor [3]. Based on this, a simple and effective tool has been created for temperature and pulse rate measurement which can be connected to the Smartphone.

\section{RELATED WORK}

The advancement in IOT has attracted a lot of researchers as well as the industry in the healthcare sector. The amalgamation of mobile communication along with IOT can be very useful in solving future health problems. A review about the acquisition of mobile health data via wearable devices is presented in [1]. The author concludes that the mhealth system based on IOT will revolutionize the way in which clinical research is conducted. There has been development in real time smartphone based mobile health systems like the one described in [2]. Their system uses a heart rate monitor to measure the heart rate in bpm. The paper describes a system which can be used to transfer heartbeat data on the patient's mobile phone. Another report authored by [4] describes a real time wireless health monitoring system to measure patient's temperature, pulse rate, blood pressure and ECG data. The author concludes that the system calculates patient's physical health data with precision.

\section{SYSTEM ARCHITECTURE}

\section{Hardware}

The hardware design consist of a microcontroller ATmega328P, AD8232 Heart monitor sensor, three-lead electrode pads, pulse sensor, LM35 temperature sensor, HC05 Bluetooth module. The hardware design is shown in Fig. 1.

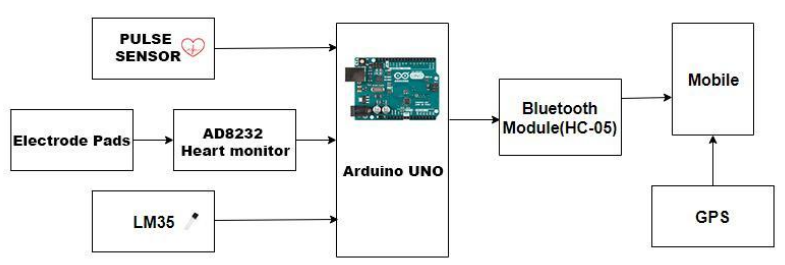

Fig.1. System architecture design

Published By:

Blue Eyes Intelligence Engineering 


\section{Microcontroller}

The microcontroller unit which was used for this project was the ATmega328P. The low power, high performance 8 bit AVR RISC-based microcontroller incorporates $32 \mathrm{~KB}$ ISP flash memory along with read-while-write capabilities. The ATmega328P is a single chip microcontroller which operates between 1.8-5.5 volts [10].

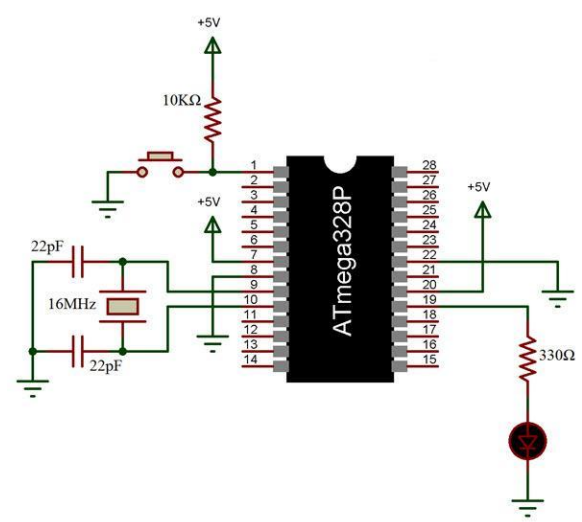

Fig.2. ATmega328p schematic diagram.

\section{AD8232 Heart Rate Monitor}

The AD8232 is a single pulse rate module front end integrated circuit used for ECG and other body signals estimation applications. It is designed to measure, amplify and filter small signals in the presence of noise. The pulse rate module provides an ultralow power analog-to digital converter (ADC) and an embedded microcontroller to obtain the output signal [2]. The AD8232 board measures electrical activity by placing electrode pads on our body. The hardware design of the ECG monitoring is shown in Fig. 3.

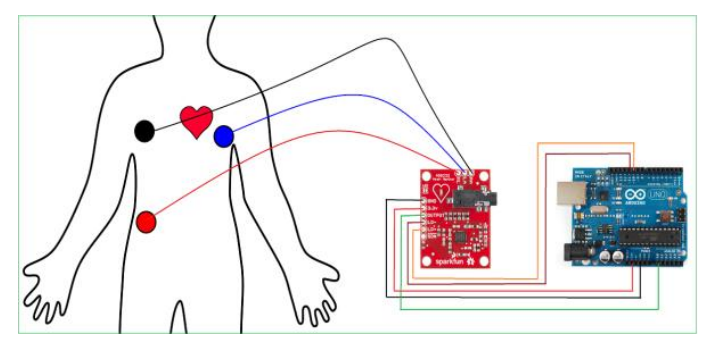

Fig.3. AD8232 Heart rate monitor experimental setup.

\section{Electrode Pads}

The pads used to get ECG signals were three conductive pads attached to the body. These cables are 24" long along with a $3.5 \mathrm{~mm}$ audio jack connector on one end with snap style receptacles for biomedical sensor pads. Each cable comes in a red/blue/black set [2].

\section{Pulse Sensor}

Pulse sensor is a device which can be plugged to the body and can be used by anyone to measure pulse rate in beats per minute [9]. The sensor has two sides on one side the LED is placed and on the other side some circuitry. The pulse sensor works on the principle of optoelectronics. The heart beat is measured due to the difference in the volume of blood which causes a change in the light intensity through any organ. The LED on the front side of the sensor is placed on fingertip or ear tips.

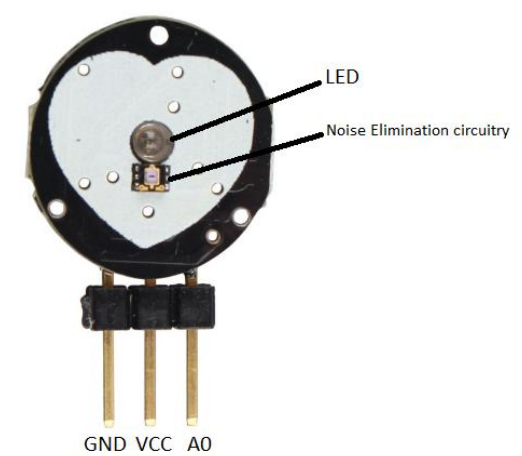

Fig.4. Pulse sensor

\section{LM35 Temperature Sensor}

The temperature of a person's body can be measured by applying LM35 to the skin surface after reaching a stable state. The sensor can be used in numerous applications for example, child incubators, patient monitoring and medical research labs. The electrical output of LM35 temperature sensor is proportional to degree Celsius. For measuring the body temperature, the left pin of LM35 is connected to power supply of $5 \mathrm{~V}$, the middle pin is the output and the right pin is connected to ground.

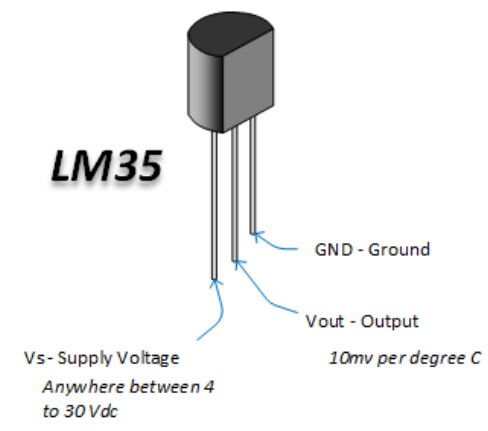

Fig 5: LM35 Temperature Sensor

\section{HC-05 Bluetooth Module}

The HC-05 is a Bluetooth module that is designed to connect to various devices via a wireless communication setup. The Bluetooth module is used to communicate between arduino and other devices like mobile phones, laptop etc. The HC-05 allows switching mode between master and slave mode.

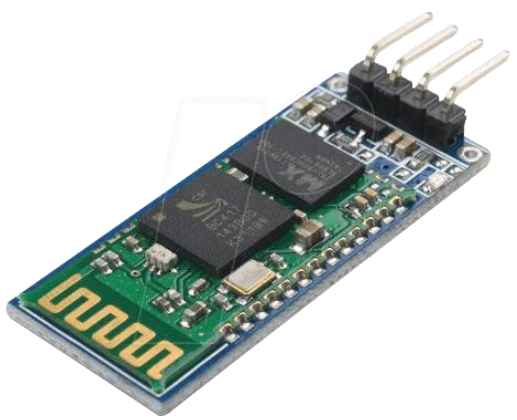

Fig.6 HC-05 Bluetooth module

Published By: 


\section{Smartphone App}

The application was designed using MIT App Inventor 2. MIT App Inventor 2 is an application that allows us to build apps using programming illustration. It is an open-source web application which uses graphical interface and blockbased coding programs which allow users to create applications that can be used on smart phones. Once the app is created it starts by selecting the Bluetooth module. When Bluetooth is connected, buttons will appear which can be selected by the patient to read the data from the ECG sensor, pulse sensor and temperature sensor. After selecting the required sensor the app will extract data from the arduino and show it on the screen. Fig.7. shows the programming logic to receive sensor readings.

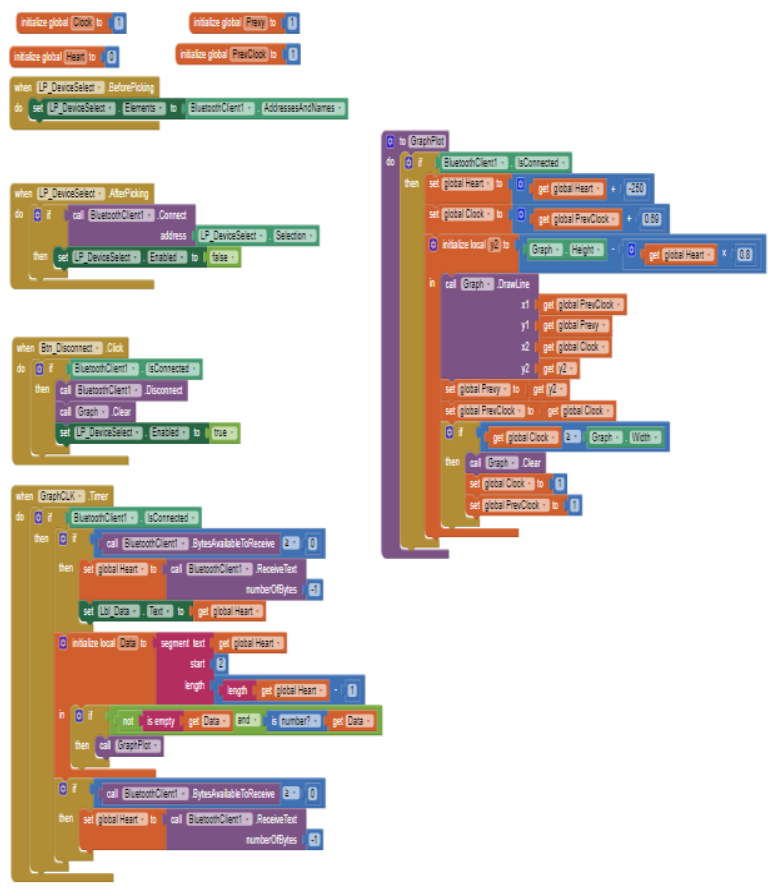

Fig.7. Smartphone App for Temperature Sensor, Pulse sensor and ECG sensor.

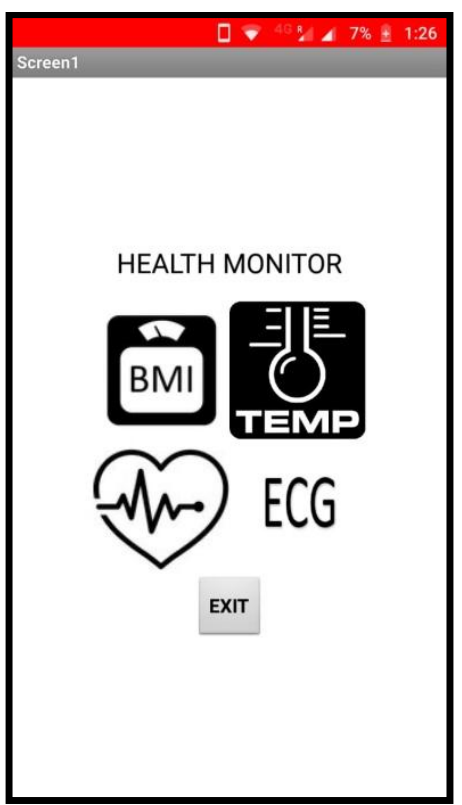

Fig.8. M-health android App

\section{RESULT}

The module created for this project is a hand device as shown in Figure 10. A Smartphone app, created using MIT App Inventor 2 was used to create the mobile app which monitors the BMI, pulse rate as well as the temperature of the patient. The Smartphone app is shown in figure 7.The Smartphone app starts working when the HC-05 Bluetooth module is selected. Once the Bluetooth is connected, after 10 seconds the app starts reading the data. Figure 9(a) is the view when the user is required to enter the weight and height for calculating the BMI. Once the button is pressed the app shows the BMI of the person and also analyzes it After calculating the BMI, the temperature sensor button is pressed. Figure 9(b) is the view when the app displays the temperature of the person. The temperature of the person is monitored continuously. Next the pulse sensor button is pressed. Figure 9(c) is the view when the app displays the heart rate of the person in beats per minute. Lastly, ECG sensor button was pressed. Figure $9(\mathrm{~d})$ is the view which displays the ECG graph of the person under observation. Overall our program and app worked properly and accomplished all goals proposed by our design.

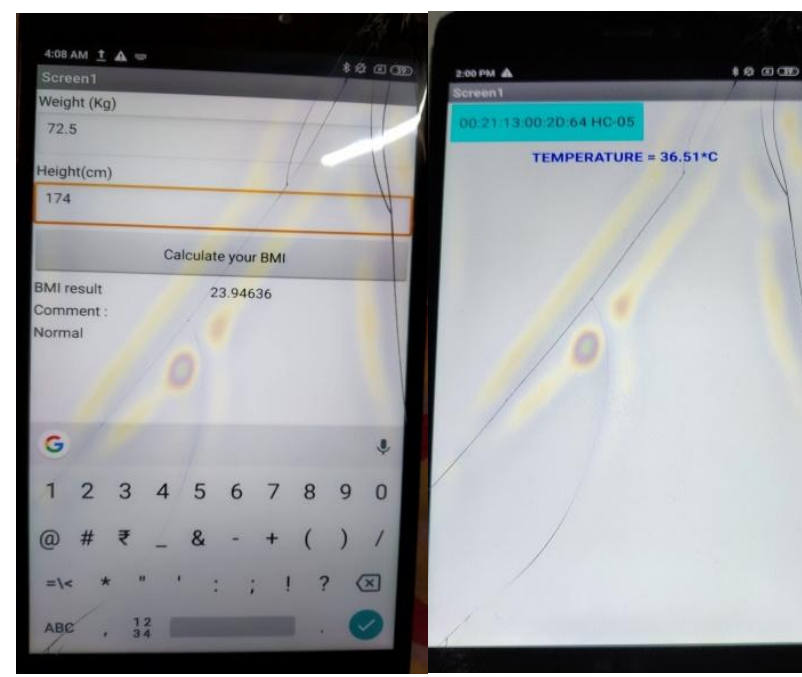

(a)

(b)

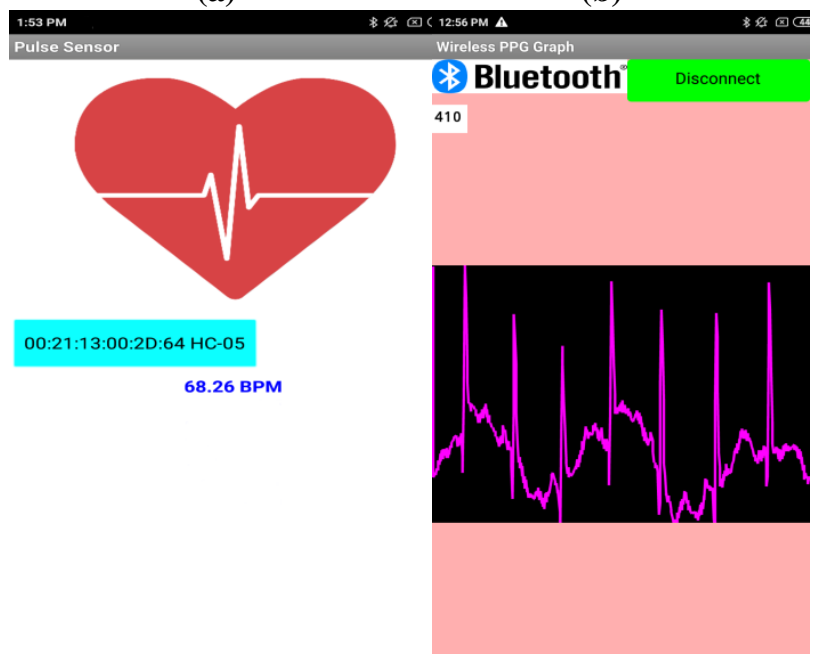

(c)

(d)

Fig.9. Android Application Display. 
The smart health aid model incorporates three system module for collecting information, storing data and accessing person health information to the doctor. The designed model made by combining Arduino uno microcontroller with Wireless Body Sensor Network. The model uses Temperature sensor for body temperature, Pulse sensor for Blood pressure count, ECG sensor for Heart monitoring. Sensors are connected with different body parts which help in getting the health information without interfering the daily routine of the person. This information containing the health of the person is transferred to physician's server using Mobile technology at any point of time.

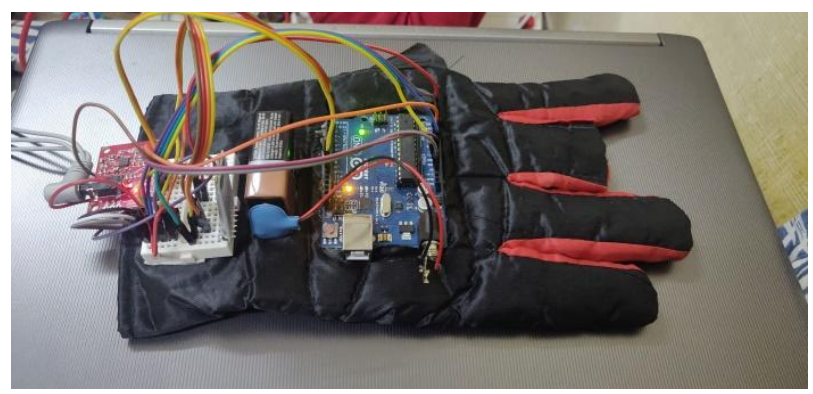

Fig.10. Experimental setup for acquiring a pulse, temperature, ECG signal

A sample report of a patient is shown in Table 1. The report contains personal information about the patient along with heart rate and body temperature of the patient. The report shows a comparison between the normal values and the calculated result. According to the data calculated it can be analyzed that the same results were obtained as that of the normal values of heart rate and temperature from our system. The ECG graph is not much clear due to the noise generated by electrical components used in the project. Also another issue with the system was the efficiency of pulse rate monitor while detecting the readings from the patient. Due to the basic design of the sensors the light generated from the pulse sensor is sometimes not transmitted properly thus readings cannot be obtained easily. Also while calculating the body temperature there is a minor variation in the actual body temperature due to the presence of surroundings. Another major concern with the project is security because Bluetooth allows anyone to connect to the application and thus get information about the patient. However if the device proves to be helpful then security issues can be alleviated.

Table 1: BMI, Heart Rate and Body Temperature of the patient.

\begin{tabular}{|c|c|c|}
\hline \multicolumn{3}{|c|}{ Personal Information } \\
\hline Name: Nakshatra Gautam & \multicolumn{2}{|c|}{ Date: 22/Feb/2019 } \\
\hline Sex: Male & & Time: 1:00pm \\
\hline \multicolumn{2}{|l|}{ Hight: $174 \mathrm{~cm}$} & Weight: $72.5 \mathrm{~kg}$ \\
\hline \multicolumn{3}{|c|}{ Normal Values } \\
\hline BMI & Heart Rate & Body Temperature \\
\hline$(18.5-25)$ & (60-100) BPM & $37.4^{\circ} \mathrm{C}$ \\
\hline \multicolumn{3}{|c|}{ Test Results } \\
\hline BMI & Heart Rate & Body Temperature \\
\hline 23.9 & $68 \mathrm{BPM}$ & $36.5^{\circ} \mathrm{C}$ \\
\hline
\end{tabular}

\section{CONCLUSION}

In this project a Smartphone based mobile health monitoring setup has been presented. In mobile health system data is recorded and transferred through wearable gadgets. The patient information can be accessed by the doctors to monitor, diagnose and advice patients from any location at any time. It is a simple system consisting of sensors connected with a Smartphone. The system can be easily used and is fast as well as cost efficient. The system can also store data for future health analysis. In this project, the app performs real-time heart rate estimation, temperature calculation and also shows ECG graphs of the patient. At the end it can be concluded that although the project is a very basic design but with more advanced products the project can work more effectively.

\section{REFERENCES}

1. Sultan H. Almotiri, Murtaza A. Khan, Mohammed A Alghamdi .: Mobile Health (m-Health) System in the Context of IoT. 2016 IEEE 4th International Conference on Future Internet of Things and Cloud Workshops (FiCloudW), pp 39 42, Aug. 2016.

2. Justin Turner, Chase Zellner, Tareq Khan, and Kumar Yelamarthi .: A Smartphone based Continuous Heart Rate Monitoring System. IEEE International Conference on Electro Information Technology (EIT), May 2017.

3. A I S Hamdani, W Purnama and D Wahyudin,.: IOP Conference Series.: Materials Science Engineering 384 $012004,2018$.

4. A. Dharma Teja1, Dr. K. Srihari Rao,.: A Smart Wearable System for ECG and Health Monitoring. International Journal of Advance Research and Development, vol. 3 issue 2, pp 57 63, 2018

5. Amna Abdullah, Asma Ismael, Aisha Rashid, Ali AbouElNour, and Mohammed Tarique .: Real Time Wireless Health Monitoring Application Using Mobile Devices. International Journal of Computer Networks \& Communications (IJCNC) Vol.7, No.3, pp 13 - 30, May 2015

6. Rahul K. Kher .: Mobile and E-Healthcare: Recent Trends and Future Directions. Journal of Health \& Medical Economics ISSN 2471-9927, Vol.2 No.3:10, July 2016.

7. Syed Muhammad Waqas Shah, Maruf Pasha .: Iot - Based Smart Health Unit. Journal of Software, Volume 12, Number 1, pp 45 - 52, January 2017.

8. Siddique Latif, Rajib Rana, Junaid Qadir, Anwaar Ali, Muhammad Ali, Imran Muhammad Shahzad Younis, Mobile Health in Developing World: Review of Literature and Lessons From a Case Study, June 2017

9. Günter Schreier, Senior Member, IEEE, Mark Schwarz, Robert Modre-Osprian, Peter Kastner, Daniel Scherr, and Friedrich Fruhwald, Design and Evaluation of a Multimodal mHealth based Medication Management System for Patient Self Administration, July 2017

10. MIT App Inventor http://appinventor.mit.edu/explore/ai2/tutorials.html, last accessed 2019/05/27.

11. Pulse sensor data https://www.generationrobots.com/media/DetecteurDePoulsA mplifie/PulseSensorAmpedGetting StartedGuide.pdf .

12. Microcontroller https://en.wikipedia.org/wiki/ATmega328. 


\section{AUTHORS PROFILE}

Aadeesh Jain Graduate in Electronics \& Communication Engineering from Jaypee University and Information Technology, Waknaghat, H.P, India

Shruti Sabherwal Graduate in Electronics \& Communication Engineering from Jaypee University and Information Technology, Waknaghat, H.P, India

Deepankar Mathur Graduate in Electronics \& Communication Engineering from Jaypee University and Information Technology, Waknaghat, H.P, India

Meenakshi Sood Senior Assistant Professor in the Department of Electronics \& Communication Engineering at Jaypee University and Information Technology, Solan. 